FERMILAB-TM- 2615-DI November 2015

\title{
Fermilab Testbeam Facility Annual Report - FY 2015
}

M. G. Albrow ${ }^{1}$, D. Anderson 11, A. Apresyan ${ }^{11}$, A. Artikov ${ }^{9}$ L. C. Bland 13 , A. Bornheim 11 , B. C. K. Casey $^{1}$, F. Cavanna ${ }^{1+16}$, D. Chokheli ${ }^{9}$, H. J. Crawford ${ }^{12}$, C. Dilks ${ }^{6}$, J. Duarte ${ }^{11}$, E. C. Dukes ${ }^{8}$, J. Dunkelberger $^{7}$, R. Ehrlich ${ }^{8}$, J. M. Engelage ${ }^{12}$, K. Francis ${ }^{10}$, M. J. Frank ${ }^{8}$, H. J. Frisch ${ }^{4}$, C. Gagliardi $^{5}$, C. Gatto ${ }^{16}$, R. C. Group ${ }^{1}$, Y. Guo ${ }^{14}$, S. Hansen ${ }^{1}$, A. Hocker ${ }^{1}$, M. Jones ${ }^{3}$, E. Judd ${ }^{12}$, H. Kim4, S. Los ${ }^{1}$, A. Mazzacane1, E. Oberla4, Y. Oksuzian ${ }^{8}$, C. Pena'11, C. Perkins ${ }^{12}$, J. L. Raaf1, E. Ramberg1, R. Rivera1 ${ }^{1}$, A. Ronzhin ${ }^{1}$, P. Rubinov1, M. Sergeeva7 , P. Shanmuganathan ${ }^{15}$, C. Snyder ${ }^{2}$, E. Song 8 , M. Spiropulu ${ }^{11}$, Z. Tang14, J. Trevor ${ }^{11}$, O. Tsai ${ }^{7}$, L. Uplegger ${ }^{1}$, C. Wiggin ${ }^{1}$, Y. Wu ${ }^{8}$, S. Xie ${ }^{11}$, L. Zhou ${ }^{14}$

\section{Editors: S. Geer ${ }^{1}$, M. Rominsky ${ }^{1}$, C. Tate $^{1}$}

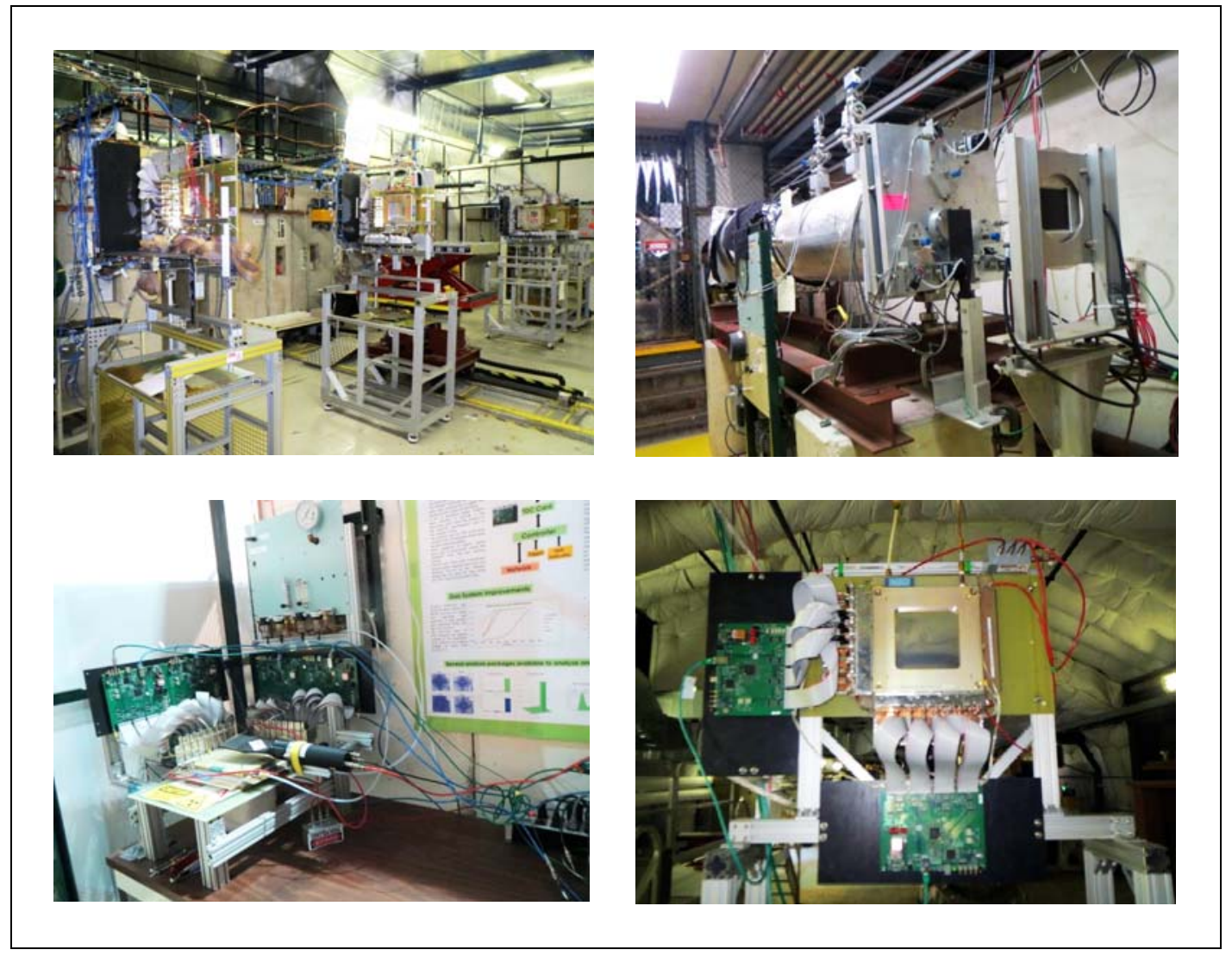

${ }^{1}$ Fermilab, ${ }^{2}$ Iowa University, ${ }^{3}$ Purdue, 4 University of Chicago, ${ }^{5}$ Texas A \& M, ${ }^{6}$ Pennsylvania State University, ${ }^{7}$ UCLA, ${ }^{8}$ University of Virginia, ${ }^{9}$ JINR, ${ }^{10}$ Northern Illinois University, ${ }^{11}$ Caltech, ${ }^{12}$ University of California-Berkeley, ${ }^{13}$ Brookhaven, ${ }^{14}$ USTC-China, ${ }^{15}$ Kent State University, ${ }^{16}$ INFN 


\section{INTRODUCTION}

This Technical Memorandum (TM) summarizes the Fermilab Test Beam operations for FY 2015. It is one of a series of annual publications intended to gather information in one place. In this case, the information concerns the individual experiments that ran at FTBF and are listed in Table TB-1. Each experiment section was prepared by the relevant authors, and was edited for inclusion in this summary.

\subsection{The Fermilab Test Beam Facility in Fiscal Year 2015}

The Fermilab Test Beam Facility (FTBF) gives users a place to characterize, calibrate, and develop new detector technologies. Since it began operation in 2005 the facility has served 73 experiments, consisting of 835 individual experimenters from 177 institutions in 30 countries. A plan view of the facility is shown in Fig. TB-1 and the website URL for the facility is ftbf.fnal.gov.

In FY15, the facility underwent a few changes. Aria Soha has moved on to other projects and Mandy Rominsky has taken over as the facility coordinator. We would like to thank Aria Soha very much for her time and dedication to running the test beam. In addition to the change in management, the following has been accomplished:

- The MCenter beam line is taking physics quality data with the LArIAT experiment.

- We have updated our website and are working on various projects both to improve the beam line and improve the building infrastructure.

- We have implemented electronic signatures for the operational readiness clearance (ORC) signoffs and computerized the controlled access log information.

- We have started implementing a facility wide DAQ

- We completed an upgrade to the facility multiwire proportional chambers and installed a cosmic telescope that is available to users.

\subsection{Future projects at the Test Beam}

Over the course of the next year, the following projects will be the primary focus at the test beam facility.

- Implementing a DAQ system that controls all the instrumentation at the test beam

- Gathering feedback from the user community on what types of instruments are needed to meet the needs of future users

- Organize a meeting of test beam users, most likely in the summer of 2016. 
Other projects that will happen over the course of the next five years include:

- Fully characterizing the beamline and implementing beam intensity monitors

- Writing analysis code for all the instruments at the test beam

- Updating infrastructure at the test beam (offices, HV systems, etc)

In addition, we'll incorporate user feedback over the next year to solidify plans for beyond FY16.

\subsection{Research Performed at the FTBF in FY 2015}

Each test-beam experiment is required to prepare a Technical Scope of Work (TSW) with the Laboratory, in which the beam, infrastructure, and safety requirements are spelled out in detail. Three new TSWs were approved in FY2015, and eight new experiments took data during FY 2015. An additional seven experiments returned from previous years to take more data in FY 2015. These 15 experiments are listed in Table-1, and represent 321 collaborators from 84 institutions in 20 countries. The chart in Figure- 3 shows the change in these numbers over the last 5 years. The FTBF was scheduled for beam for 35 weeks of the 52 week period. Beam delivery started on November 3, 2014 and continued until July 4, 2015. A total of $6.4 \times 10^{15}$ protons were delivered to MTest and $2.0 \times 10^{15}$ protons were delivered to MCenter.

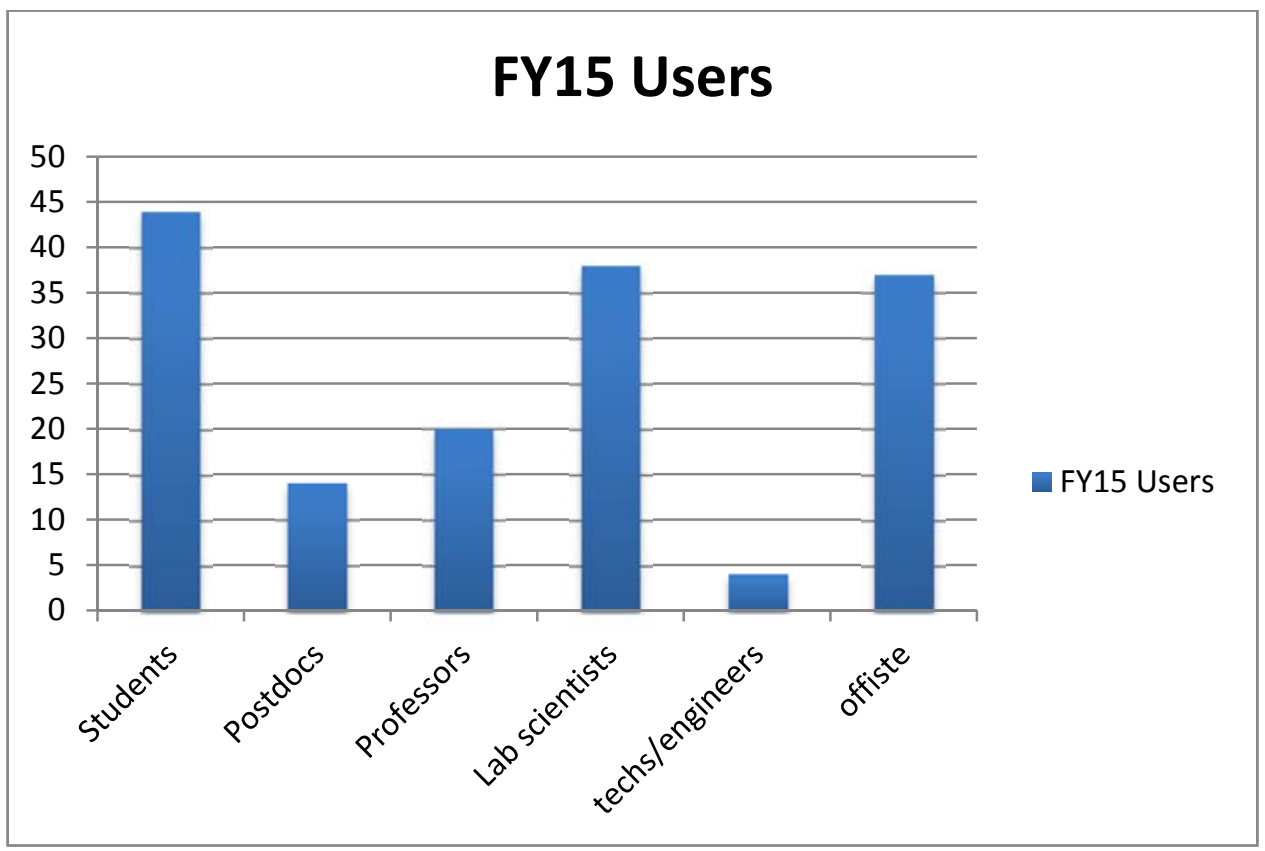

Figure 1: FY15 User Breakdown by Professional Class 
Table 1: Test Beam experiments performed in FY 2015.

\begin{tabular}{|l|l|}
\hline Test & Description \\
\hline T977 & MINERvA \\
\hline T1015 & DRC in Glasses \\
\hline T1065 & Secondary Emitters Study \\
\hline T1064 & STAR Forward Calorimeter Systems \\
\hline T1043 & Mu2e CRV Studies \\
\hline T1041 & CMS Forward Calorimetry R\&D \\
\hline T1042 & Muon g-2 Straw Tracker \\
\hline T1018 & UCLA Calorimeter Studies \\
\hline T1054 & SPHENIX PreShower \\
\hline T1058 & Secondary Emission Calorimeter \\
\hline T979 & Fast Timing Counters for PSEC \\
\hline T992 & Radiation Hard SLHC sensor tests \\
\hline T994 & JASMIN (MS1) \\
\hline T1034 & Lariat (MCenter) \\
\hline T1059 & Optical TPC (MCenter) \\
\hline Outreach & Material Studies in Nuclear Reactors \\
\hline
\end{tabular}




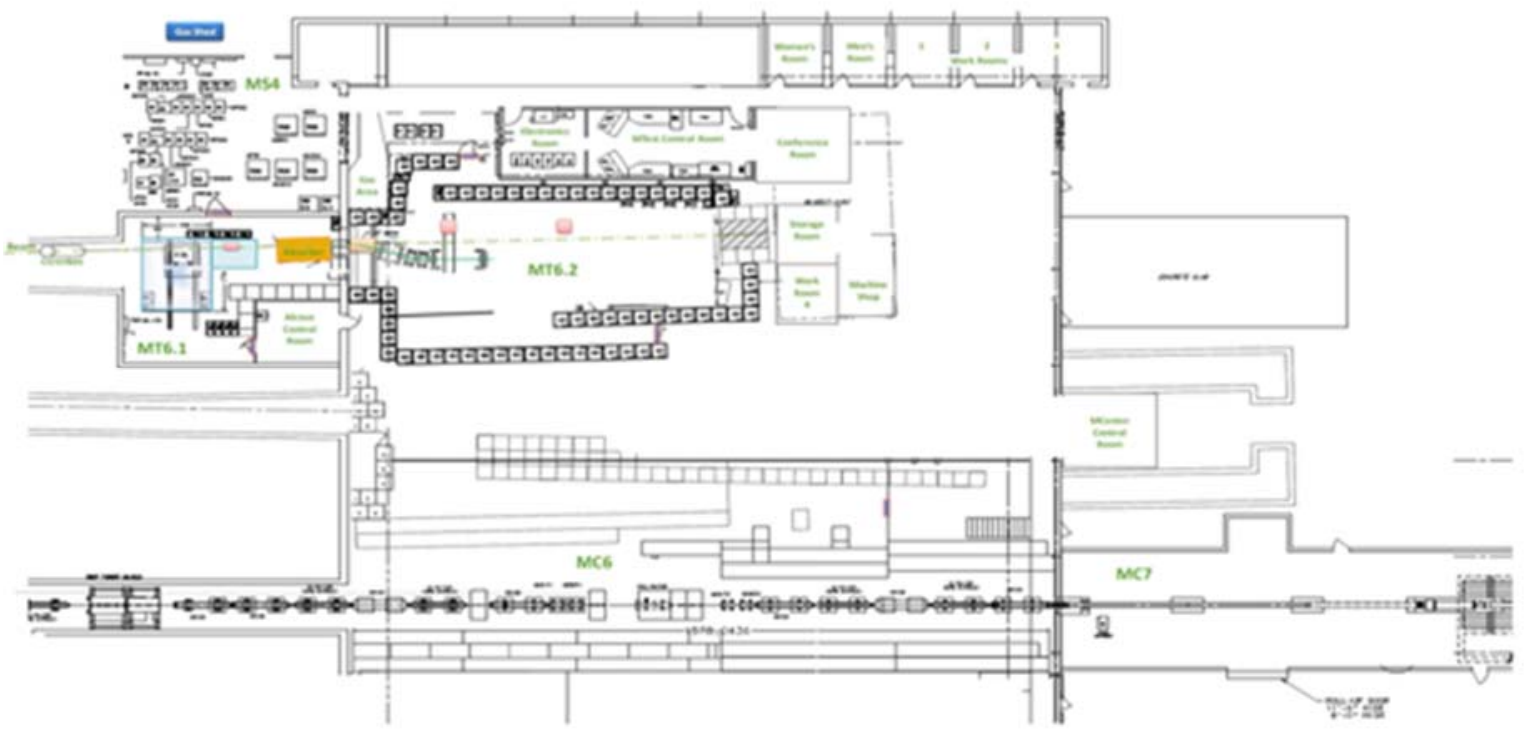

Figure 2: View of the Fermilab Test Beam Facility.

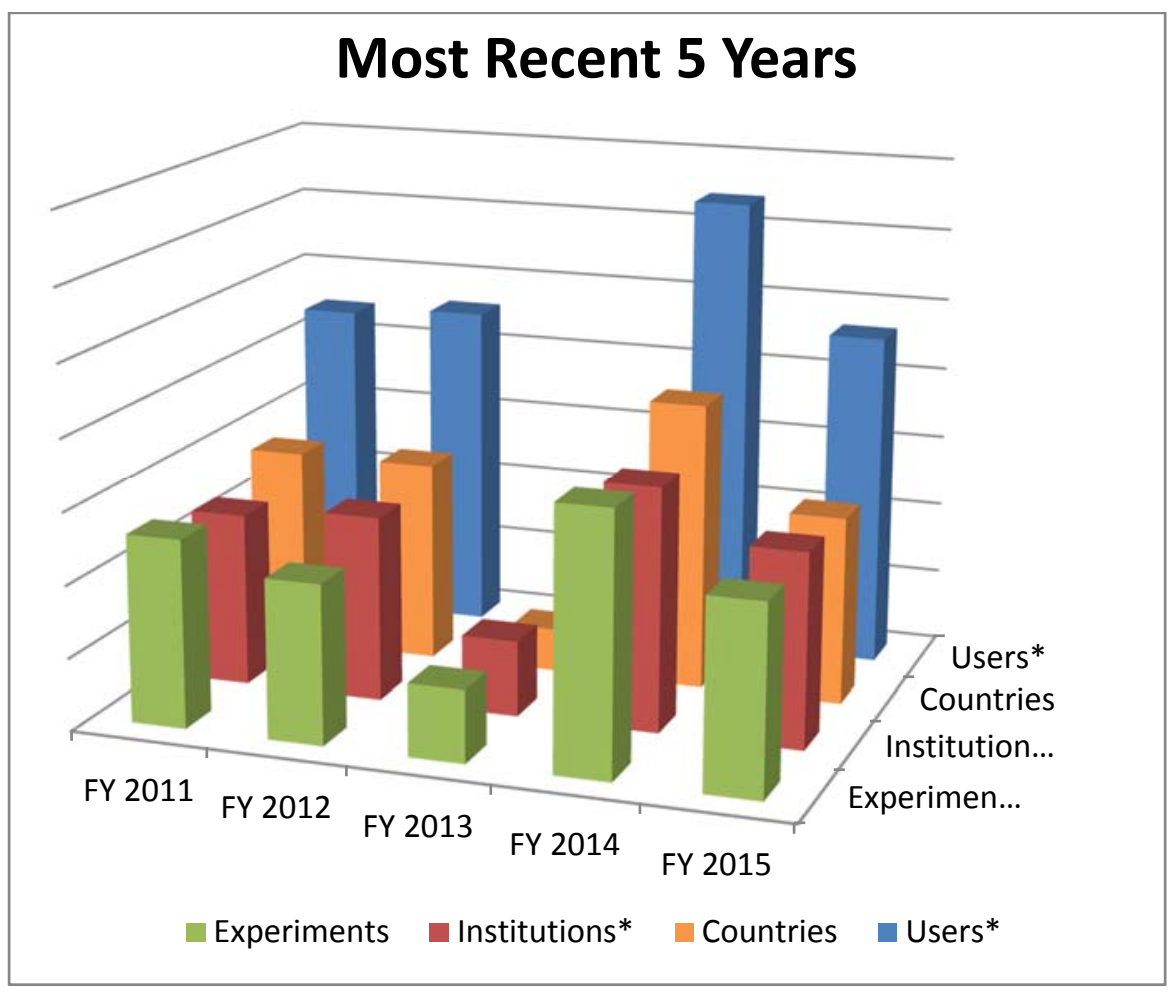

Figure 3: Growth in number of experiments, collaborators, institutions and countries served by the FTBF over the past 5 years. *Number of Users has been scaled to fit on plot. *Number of Institutions has been scaled to fit on plot. Note that due to the planned long shutdown FY12 consisted of only 7 months of beam and FY13 consisted of only 1 month of beam. 
FERMILAB-TM- 2615-DI November 2015
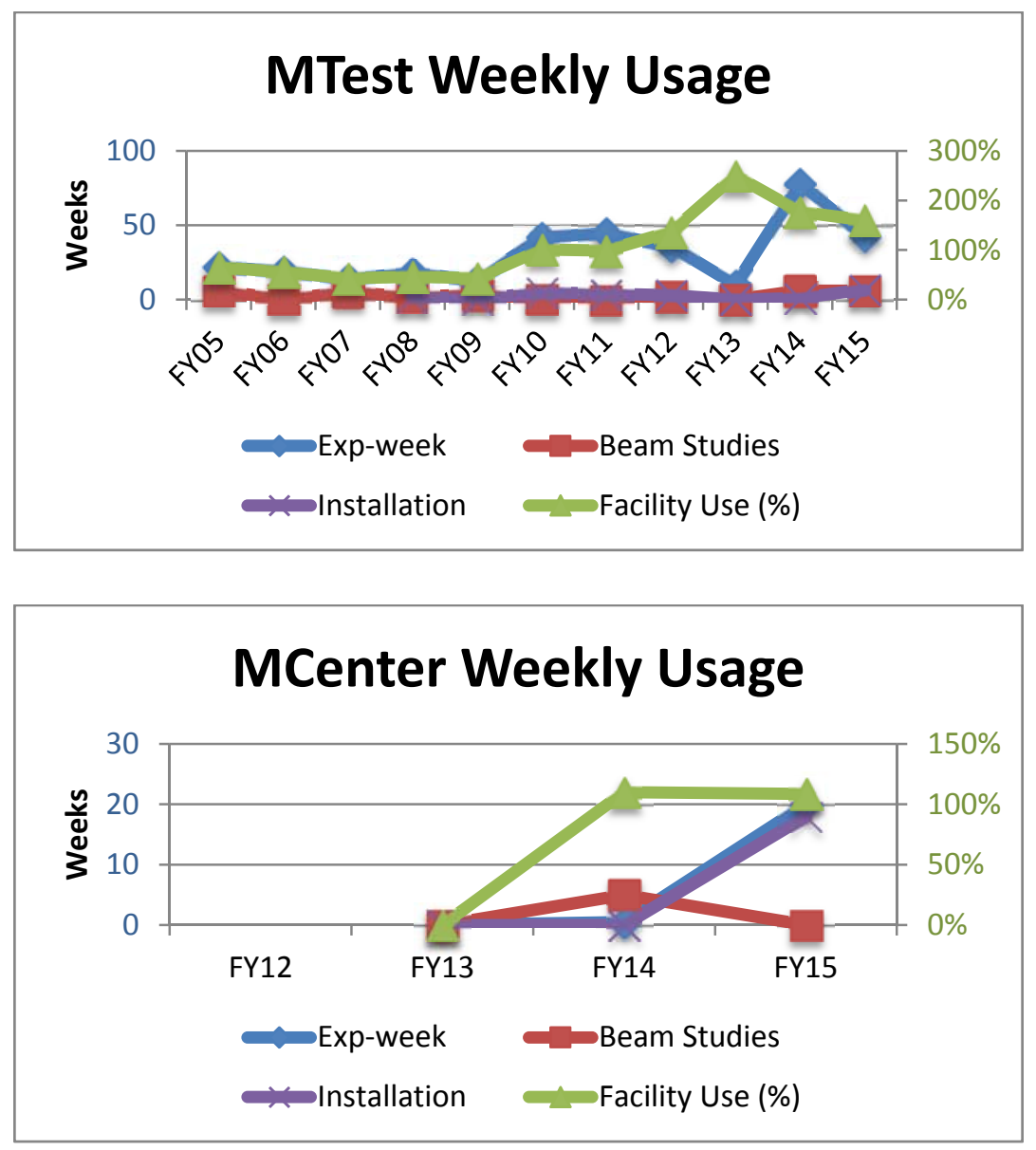

Figure 4: Weekly Usage of MTest (top) and MCenter (bottom) beamlines, nominalized to number of beam-weeks available.

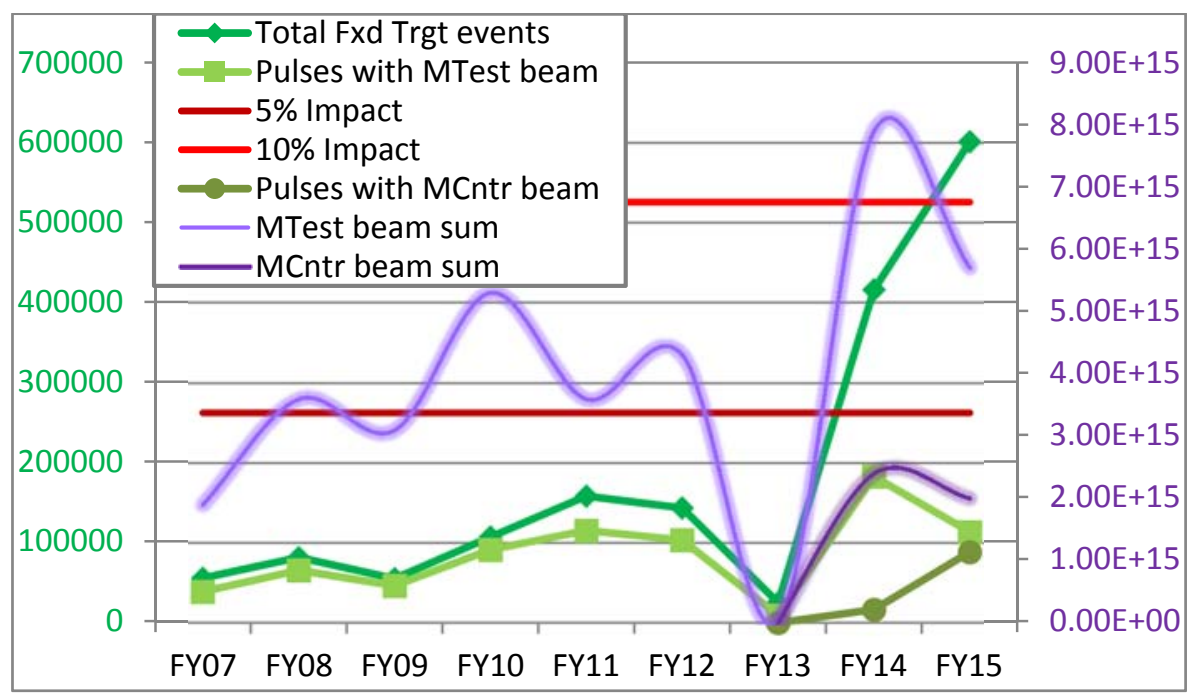

Figure 5: Fixed target pulses to FTBF. 
FERMILAB-TM- 2615-DI

November 2015

\section{SECTION 2: REPORTS FROM THE TEST EXPERIMENTS}


FERMILAB-TM- 2615-DI November 2015

T-977 (MINERvA Testbeam Collaboration)

\section{Beam used:}

Primarily low-energy pion mode beam in both polarities; some $120 \mathrm{GeV}$ protons and muons for calibration and testing.

\section{Run dates:}

Installation began in the 2014 shutdown. Recordable data runs were on 2-16, 30-31 Mar; 2-27 Apr; 5 May; 24 Jun - 3 July.

\section{Motivation and Goals:}

Measure the energy response of individual parts of the MINERvA detector to charged pions and electrons. Figure I shows the results of the MINERvA testbeam pion response study for the energy range appropriate for the neutrino spectrum in the "Low Energy" beam which was delivered up until May 2012. The (hypothetical) extension to the right of the figure shows how we now need to extend this result to higher energy spectrum of post-2013 "Medium Energy" neutrino beam.

\section{0 preliminary results and 2013 reach}

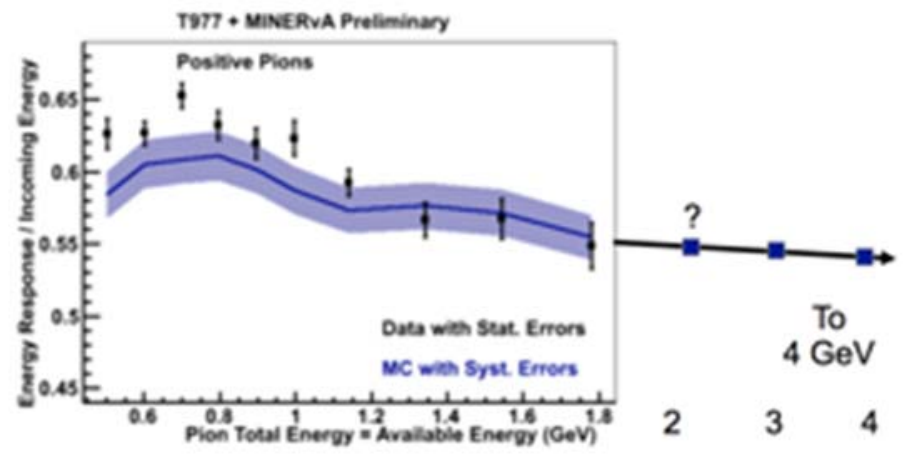

Figure I

\section{Setup:}

The facility instrumentation used pretty much as-is was: multiwire tracking chambers, and their scintillator-based trigger, helium drift tubes, lead glass block calorimeter, Cherenkov detectors, overhead crane and other mechanical support. We also made use of the Accelerator Divisions' readout of scalars as well as their logging of beamline parameters (ACNET).

Facility instrumentation that was used with substantial modification or upgrade: Time of flight system, AC power distribution.

The MINERvA testbeam detector itself, along with a veto system and the downstream time 
of flight station is in MT6.2D; the lead glass was located in MT6.2. Figure II shows the main detector. At the left of Figure II, the veto system is visible on its green Unistrut frame.

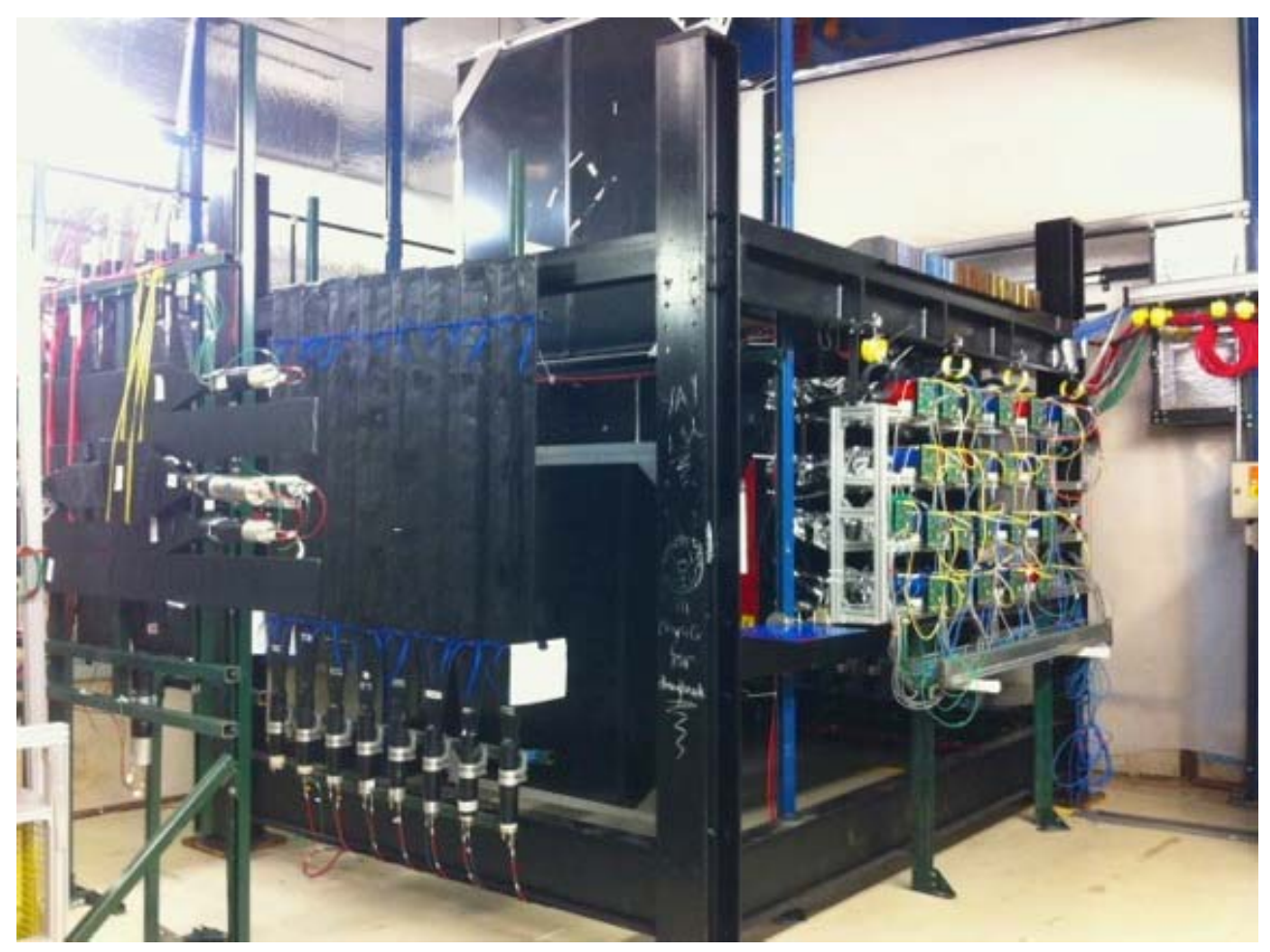

Figure II

\section{Results and Impact:}

The data is now being analyzed. The results are needed for the entirety of the MINERvA ME running scientific program; we anticipate that the analysis results will eventually be published in NIM. Another valuable achievement has been the testing of new versions of the firmware for use in the frontend electronics of the MINERvA detector. 
T-979/High Precision Timing Detectors (M. G. Albrow, E. Ramberg, S. Los, C. Wiggin, C. Snyder)

Beam used: MTest Section 2C

Run dates: June $17^{\text {th }}-23^{\text {rd }} 2015$

\section{Motivation and Goals:}

Measure signals produced by Cherenkov radiation in both quartz and sapphire bars, of a novel L-shape design, in silicon photomultipliers. Study the time resolution by comparing with a reference time (Cherenkov light and a micro-channel plate PMT) for different bar lengths. This is a 20-channel detector for an extension of CMS at the LHC to measure leading protons at $|\mathrm{z}|=220 \mathrm{~m}$. The $4 \times 5$ bar array is $12.3 \times 15.4 \mathrm{~mm}$. See drawing of front view; the SiPM array is to the left.

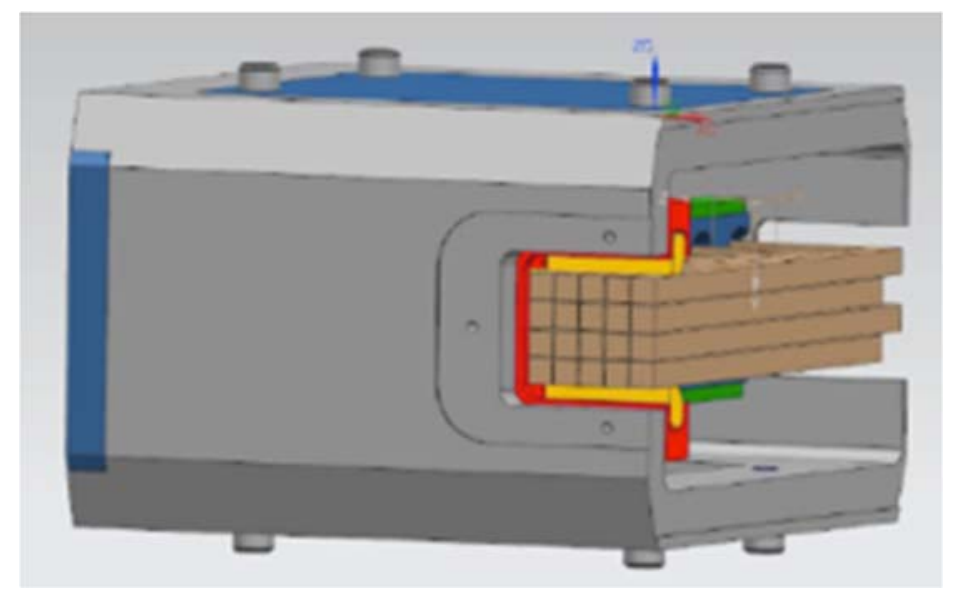

\section{Setup:}

The apparatus is set up on a moveable table in Section 2C. It consists of a (left to right in photo) small trigger counter (scintillator + PMT), the QUARTIC detector under test, a scintillator+PMT with a central hole used as a veto counter, and an MCP-PMT-240 in the beam to provide a fast reference time signal. 12 channels were read at a time by DRS-5 5 GS/sec waveform digitizers. In addition, and provided by the Fermilab Test Beam group, were some wire proportional chambers both upstream and downstream to measure tracks and interpolate them to the QUARTIC detector. 
FERMILAB-TM- 2615-DI November 2015

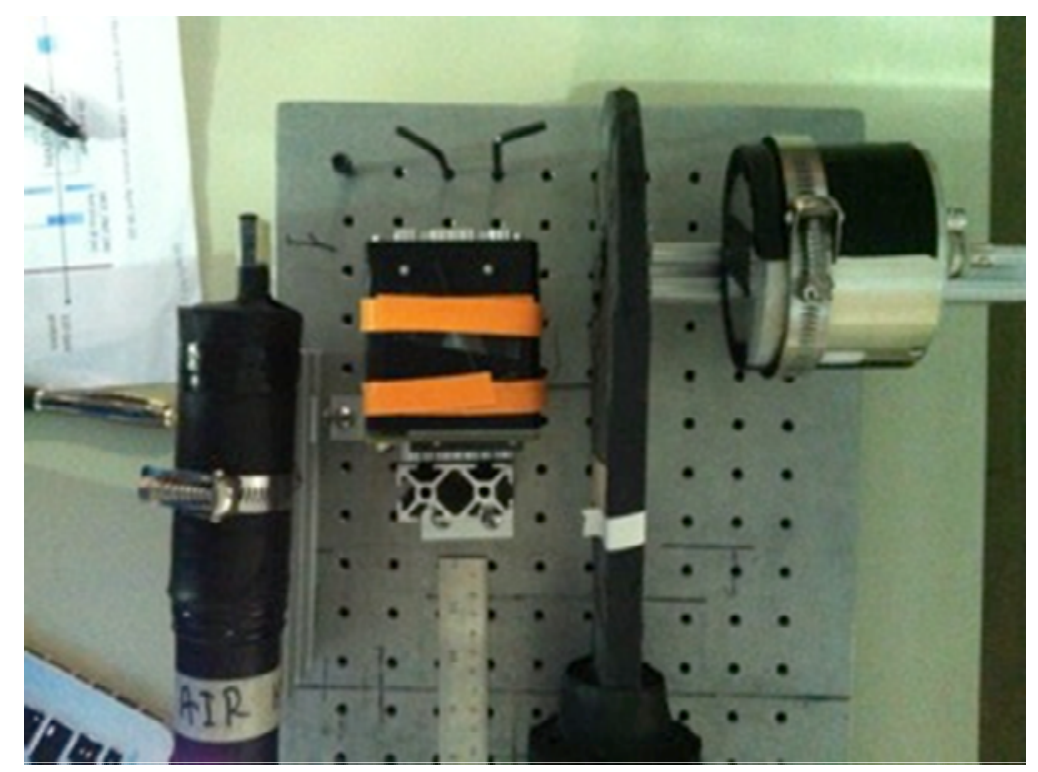

\section{Results and Impact:}

The wire chamber data enabled straight tracks to be found and interpolated to the zposition of the quartic bars, so that the signals in the hit bar (out of 20) can be analyzed. The time sample ( $200 \mathrm{ps}, 1024$ samples) with the highest signal was found, and it was very well separated from noise (from bars with no hits). The same procedure was applied to the reference time counter, and the time at which the rising signal crossed $50 \%$ of the peak was found. From the time difference distribution we extract the time resolution, after correcting for pulse-height slewing (the correlation between pulse height and time difference). The final corrections, including signals from neighboring bars, are still to be done. A few channels did not have signals, traced to poor optical contact between bars and SiPMs, which will be corrected with a clear epoxy joint. It was demonstrated that the design is sound and this new type of quartz Cherenkov counter can be (and will be) used in CMS at the LHC for an extension of the physics program.

A paper will be submitted to J. Inst. when the results are final. 
T-992 / Tests of radiation-hard sensors for the SLHC (R. Rivera, L. Uplegger, M. Jones)

Beam used: $120 \mathrm{GeV}$ protons

2015 Run dates: Mar 24-30, Jun 3-23

Motivation and Goals:

At the SLHC, after $2500 \mathrm{pb}^{-1}$ of data, the Expected maximum fluence for the pixel region $(<20 \mathrm{~cm})$ will be $2.5 \mathrm{x}$ $10 \mathrm{E} 16 \mathrm{~cm}^{-2}$. To cope with this unprecedented radiation environment, there have been quite a few international collaborations formed to find possible solutions for vertex and tracking detectors at the SLHC. These include the RD42, RD49, and RD50

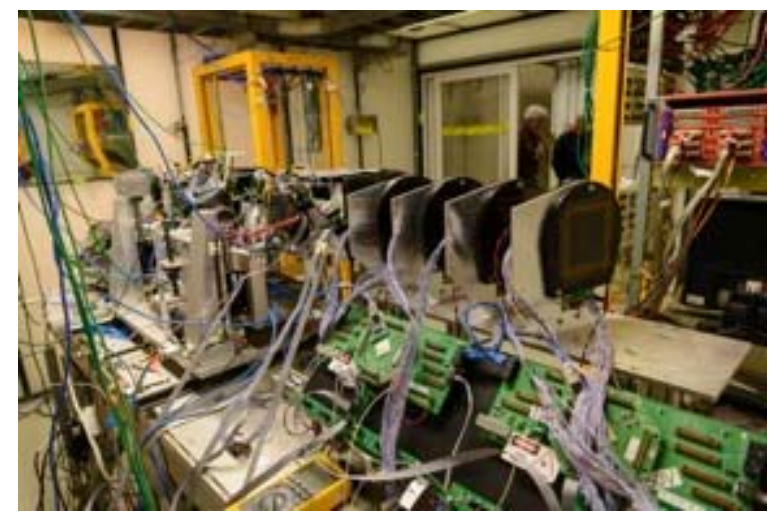

Figure 1 In situ T-992 test beam setup collaborations. A variety of solutions have been pursued. These include diamond sensors, 3D sensors, MCZ planar silicon detectors made from MCZ wafers, epitaxial, p-type silicon wafers and thin silicon detectors. The experimenters wish to compare the performance of this wide variety of detectors in a test beam before and after irradiation. To do so, the experimenters use the FTBF pixel and strip tracking telescopes which have $\sim 8 \mu \mathrm{m}$ resolution at the device under test. In particular, the experimenters are planning to study the charge collection efficiency of irradiated and un-irradiated devices and the spatial resolution as a function of the track incident angle. The experimenters will change the incident angle of the beam by moving the sensors, to investigate how the resolution varies with angle. Many physicists participating in this beam test are members of the RD42 and/or RD50 collaborations.

\section{Setup:}

The pixel and strip telescopes are read out through a custom DAQ system known as CAPTAN. A gigabit Ethernet board is used to route UDP data to a computer which is connected to a Fermilab server via internet. The readout boards are located close to the detector in the hut, and share a common clock and trigger signal. The detectors themselves may be operated up to $\sim 800 \mathrm{~V}$. No exposed HV parts are present.

\section{Results and Impact:}

We have studied both 3D silicon and diamond sensors and new prototypes of planar sensors with a small pitch $(25 \mu \mathrm{m})$. 3D tracking detectors are promising radiation-hard candidates to replace planar detectors in the HL-LHC. Radiation damage effects are measured with regards to charge collection, efficiency, and resolution of the particle tracks in beam tests, as well as leakage current and pixel noise. We have studied three varieties of $3 \mathrm{D}$ sensors: $1 \mathrm{E}, 2 \mathrm{E}$ and $4 \mathrm{E}$ (the number referring to the number of implant pattern per 
FERMILAB-TM- 2615-DI November 2015

pixel). After irradiation, the 2E showed the least degradation in efficiency and collected charge. These devices were provided by CNM and SINTEF. More work must be done to reach radiation hardness of $10 \mathrm{E} 16 \mathrm{neq} / \mathrm{cm}^{2}$. For diamond sensors, we have comparatively studied the tracking performance of a polycrystalline diamond mounted on the new digital Read Out Chip that allow to reach lower threshold ( $\left.2000 \mathrm{e}^{-}\right)$thus improving the efficiency of these devices that have a signal of only about $5000 \mathrm{e}$. With the new ROC we have been able to achieve efficiencies of $\sim 95 \%$.

We also measured the properties of new prototypes with a small pitch $25 \mu \mathrm{m}$. These small pitch pixels performed well and showed interesting properties of charge sharing between pixel cells that are tightly spaced. In Figure 2 we show the charge deposited in a group of 4 pixel cells.

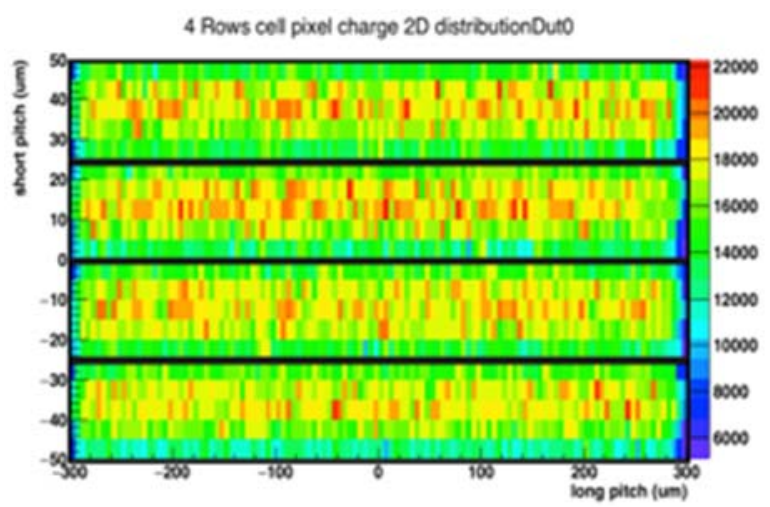

Figure 2 Charge measured in the small pitch devices 
T-1015 / Dual-readout calorimetry with heavy glasses (C. Gatto, A. Mazzacane)

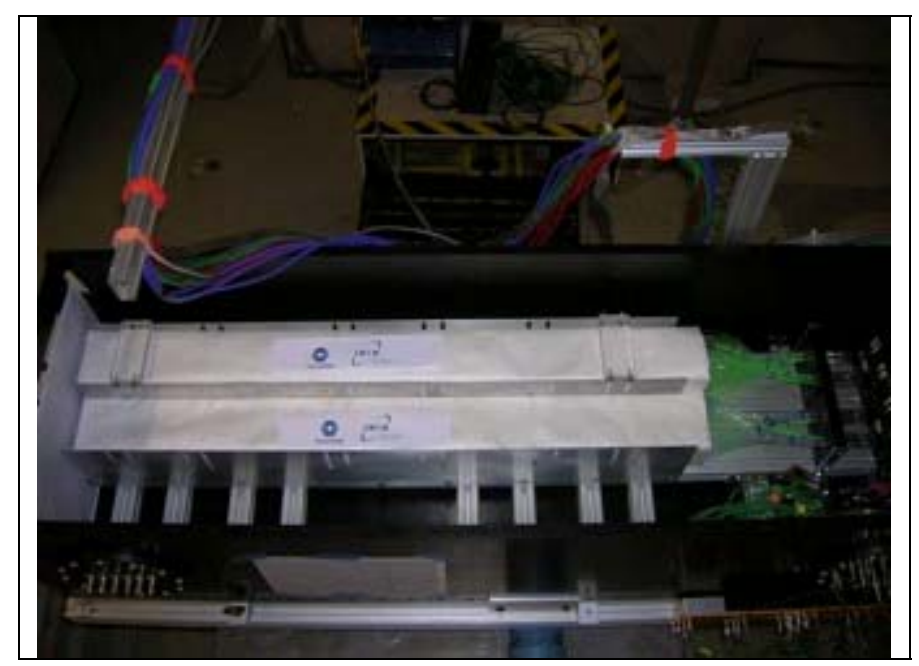

Figure 1: ADRIANO prototypes for an ILC experiment

Beam used: $120 \mathrm{GeV}$ protons, $32 \mathrm{GeV}$ muons, secondary from $0.5 \mathrm{GeV}$ to $12 \mathrm{GeV}$ Run dates: June 29, 2014-July 15, 2014; Aug 21, 2014-Sept. 4, 2014; Nov 17, 2014-Dec. 15, 2014

\section{Motivation and Goals:}

The ADRIANO technology (A Dual-readout Integrally Active Non-segmented Option) is extensively being developed as part of the research program of T1015 Collaboration for experiments at future lepton colliders or at fixed target with high intensity beam.[1] New construction techniques have been exploited and several detector prototypes have been assembled and tested at FTBF since the inception of T1015 Collaboration to study their performance. The ADRIANO, technology, initially devised to improve the performance of hadron calorimeters through the mechanism of dual-readout compensation, has been extended for applications to electromagnetic calorimeters. The fast response of ADRIANO, along with its intrinsic particle-id features, make this technology particularly well suited for future High Intensity frontier experiments

\section{Setup:}

The FTBF facilities used during the experiment are listed:

1. Pick-up for the transportation of the detector to F-Test

2. Crane for the installation of the detector

3. Remotely controlled moving table $2 \mathrm{~B}$

4. Remotely controlled rotating table

5. One wire chamber

6. Cherenkov based PID system

7. HV power supply and distribution systems (COW). 


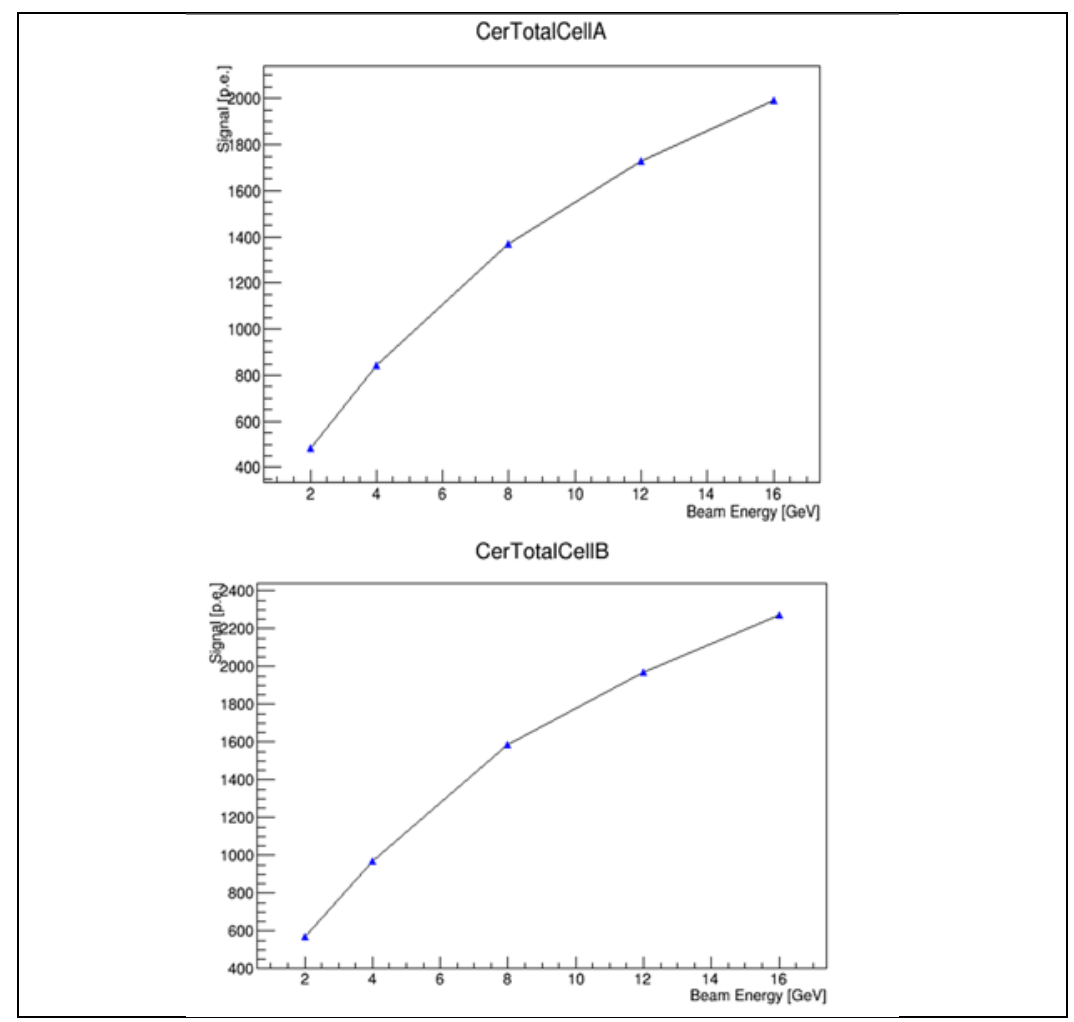

Figure 2: Energy scan of ADRIANO 2014A and 2014B

\section{Results and Impact:}

Light yield has been measured for a total of four ARIANO prototypes. The values obtained range from 340 p.e./GeV (for the high energy versions of the detector) to 4000 p.e./GeV (for the high intensity versions of the detector). These values meet the minimum requirement for use of ADRIANO in high precision experiments at lepton colliders (i.e ILC or CLIC) and lower energy experiments like ORKA or REDTOP. The results are shown at the following two international conferences:

1. CALOR2014, Giessen (Germany) Nov. 6-Nov 12014

2. LCWS2014, Belgrade (Serbia) Oct. 6-Oct 102014

3. LCWS2015, Vancouver (Canada) Nov. 2-Nov 62015 
T-1018 /(C. Gagliardi, C. Dilks, J. Dunkelberger, M. Sergeeva and O. Tsai)

Beam used: $1 \mathrm{GeV}-12 \mathrm{GeV}$ electrons, $120 \mathrm{GeV}$ protons

Run dates: May 20 -May 29, 2015

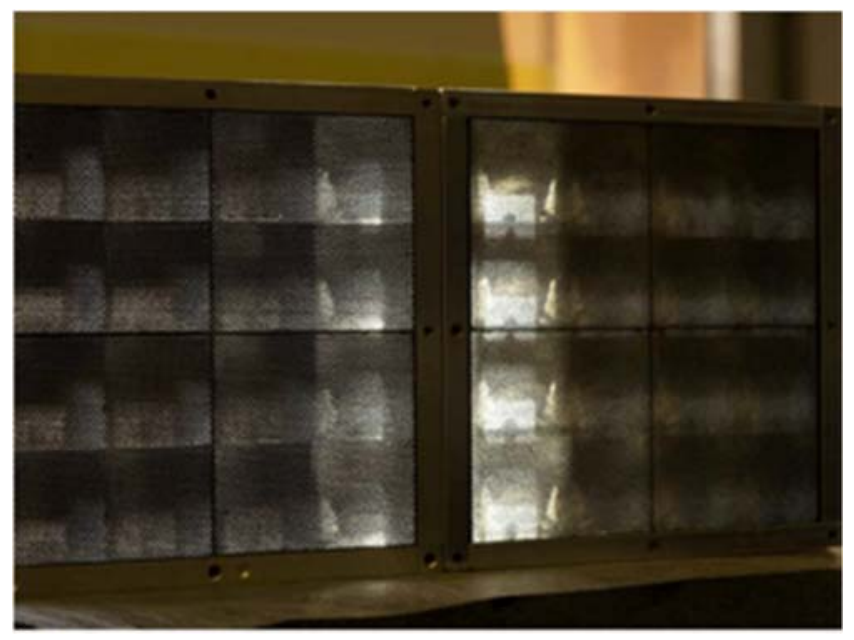

Figure 1: Two EMCal prototpyes from the side opposite to readout. The new EMCal prototype has much finer sampling frequency compared to the old prototype.

\section{Motivation and Goals:}

We are continuing development of $\mathrm{W}$ powder ScFi technology for sampling calorimeters at RHIC and EIC. In 2015, our main goal was to investigate options for a high-resolution EMCal in the outgoing electron direction for an EIC detector. In the first year of this program we planned to build a new EMCal prototype (depicted on the right side in Fig. 1) to learn the limitations of the technology and test it with the beams at FNAL. Additionally, we tested a modified light collection scheme for an EMcal prototype that we tested at FNAL last year. This was a third test run of the T1018 proposal.

\section{Setup:}

We used the MT6.2-B area for the experimental setup. Instrumentation provided by the test beam facility included a Lead Glass calorimeter, movable table $2 \mathrm{~B}$, alignment laser system, Cerenkov counter, CAMAC and NIM crates, some signal cables, patch panels, cameras and helium tubes to reduce multiple scattering. In addition we used the MWPC tracking system as a stand-alone system for monitoring beam conditions (not in our data stream). 


\section{Results and Impact:}

The energy resolution of the new EMCal prototype shown in Fig. 3 is only slightly better then for the old EMcal prototype tested in 2014. The big difference (red and green curves) between MonteCarlo and test beam data are under investigation now. The new prototype is currently being re-worked for the upcoming test run in 2016. In addition, parameters of the next version of the high resolution EMcal are now being optimized with MC. The modified compact light collection scheme worked as expected.

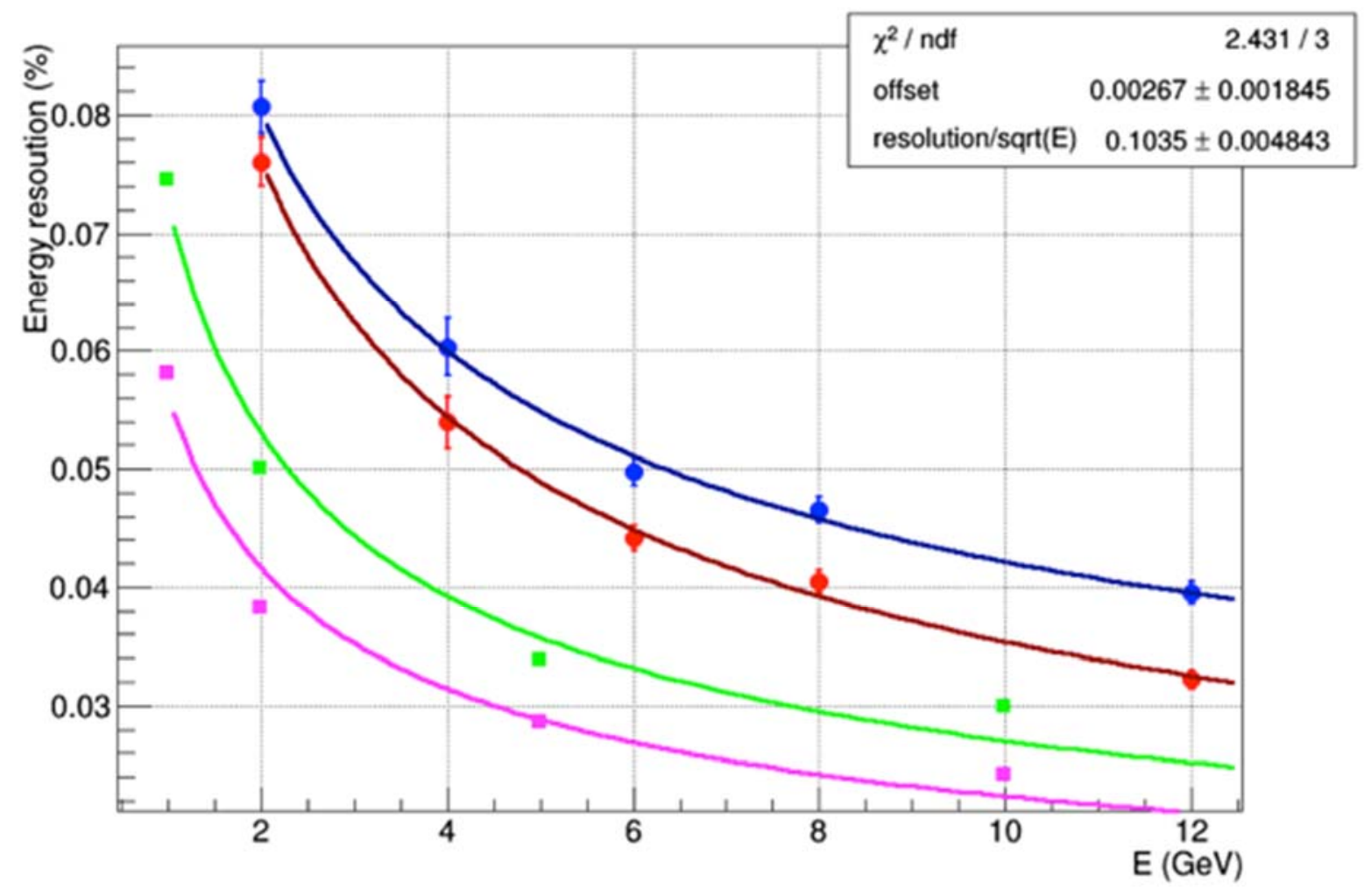

Figure 2: Energy Resolution in EM prototype compared with MC prediction.

T1018 presented results in two CALOR conferences in 2012 and 2014. Publications:

1. Journal of Physics: Conference Series 404 (2012) 012023, O. D. Tsai, et. al., 'Results of R\&D on a new construction technique for W/ScFi Calorimeters'.

2. Journal of Physics: Conference Series 587(2015) 01205, O. D. Tsai, et. al., 'Development of a forward calorimeter system for the STAR experiment.' 
T-1034/LArIAT （F. Cavanna, J. L. Raaf)

Beam used: $8-60 \mathrm{GeV} \pi^{+/-}$

Run dates: May 1 - July 3, 2015

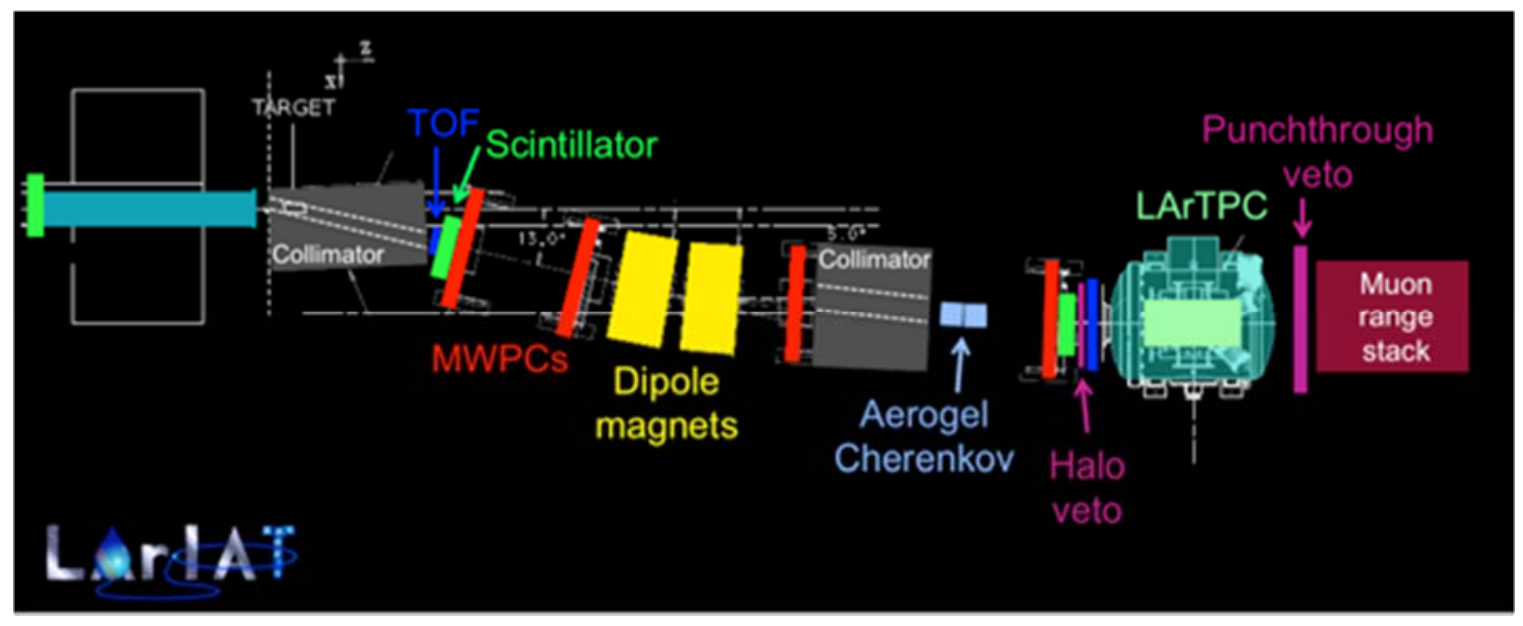

Figure 1: LArIAT layout in MCenter beamline

\section{Motivation and Goals:}

LArIAT (T-1034) will characterize the performance of liquid argon time projection chambers (LArTPCs) in the range of energies relevant to short- and long-baseline experiments such as MicroBooNE, the SBN program, and DUNE. Measurements made in LArIAT aim to be useful for both neutrino physics and for proton decay searches. Measurements of charged pion and kaon interaction cross sections will be made, and these data will also be used to optimize particle identification capabilities. In addition, LArIAT will study energy resolution improvements that may be achievable by combining information from scintillation light and ionization charge signals. The data collected in LArIAT will also be used to study the possibility of determining the sign of charged particles (pions/muons) without a magnetic field.

\section{Setup:}

The LArIAT setup is shown in Figure 1, located in the MCenter beamline at MC7. The incoming secondary beam was operated in both positive and negative polarity for a range of beam energies, and the tertiary magnets were also operated across their full range. The setup uses 4 multiwire proportional chambers (MWPCs) and 2 time-of-flight (TOF) scintillator paddles provided by FTBF. Additional beam instrumentation, such as aerogel Cherenkov counters, halo and punchthrough veto scintillator paddles, and muon range stack were put in place by the experiment. The heart of the experiment is a two-plane (240 wires each, $4 \mathrm{~mm}$ spacing) liquid argon time projection chamber (LArTPC) with 2 cryogenic PMTs and 3 SiPMs viewing the active volume. 


\section{Results and Impact:}

Analyses of LArIAT beam events are underway, with preliminary results expected before the end of the year. An initial hand-scan of a small fraction of the events shows rich features, as exhibited in the $\pi^{+}$charge-exchange interaction candidate shown in Figure 2. We are making good progress on automated reconstruction of tracks through

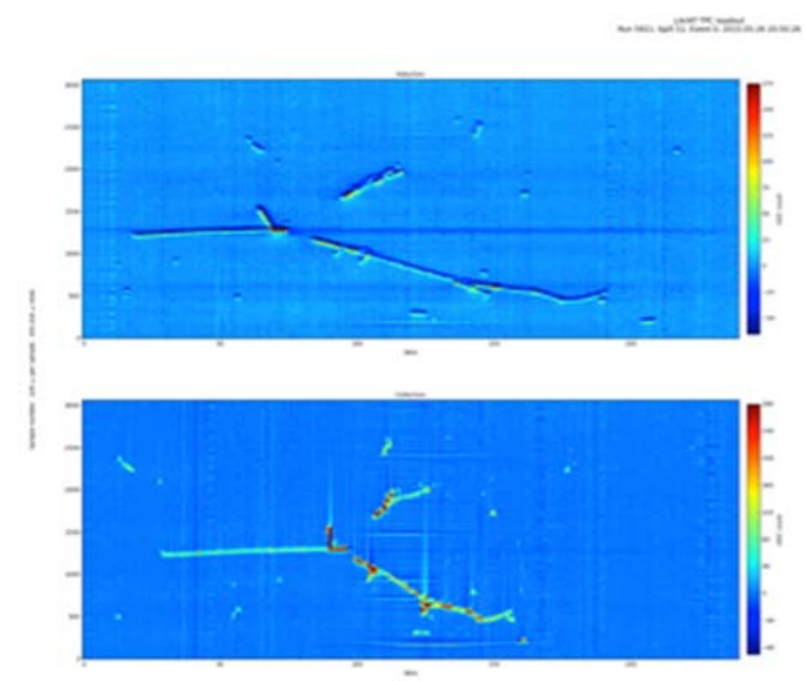

Figure 2: Candidate for $\pi^{+}$charge exchange to $\pi^{0}$ with 2 protons emitted.

the beamline wire chambers and matched into the LArTPC. Analyses of pion-Ar interaction cross sections are underway as well; these will be the first measurements ever made for pion interactions on argon nuclei. The cross section measurements will be useful for improving and validating Geant- 4 simulations, which can then be used for all future LArTPC experiments.

These data will also be used to refine particle identification techniques in LArTPCs. The results will feed back to future LAr experiments, where a better understanding of the capabilities and efficiencies for identifying different particle species will lead to improved measurements of neutrino interactions. 
The light collection system in LArIAT is another source of active analysis.

Figure 3 shows a preliminary measurement of the muon lifetime made with Michel electron candidates from muon decay. A light-based trigger was used to collect these events. Eventually, these events will be used as an energy calibration source and to make a measurement of the $\mu^{-}$nuclear capture rate. We aim to demonstrate the utility of light + charge based calorimetric energy reconstruction for improved resolution.

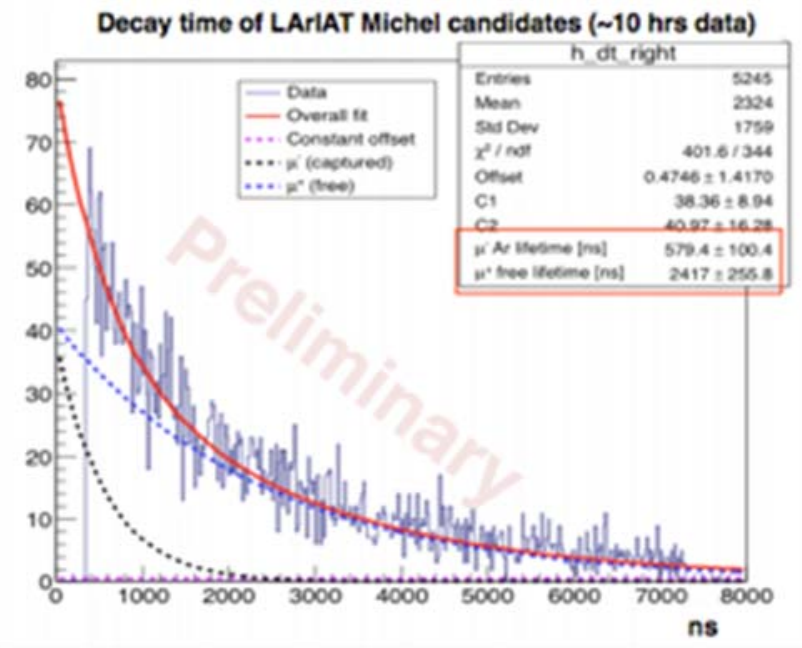

Figure 3: Muon lifetime measurement via light collection system. 
T1042/Muon g-2 Straw Tracker (B. C. K. Casey)

T1042 is a test beam experiment to validate the performance of straw trackers for the Muon g-2 experiment. In FY2015, we requested and received two weeks of data from June $3^{\text {rd }}$ to June $16^{\text {th }}$ for 12 hours a day. We measured the straw efficiency, signal to noise, and resolution as a function of gain $\left(10^{4}-10^{6}\right)$, electronics threshold, gas composition (80:20 $\mathrm{Ar}: \mathrm{CO}_{2}$ and 50:50 Ar:Ethane), and external pressure (ATM to $10 \mathrm{mTorr}$ ). We took data with $120 \mathrm{GeV}$ protons at rates ranging from $10 \mathrm{k}$ protons per fill to $100 \mathrm{k}$ protons per fill.

Our apparatus consisted of a 4 layer, 128 channel, straw system inside a vacuum chamber, 4 layers of silicon to measure individual proton trajectories, and a scintillator to determine t0. We also made use of the facility's wire chambers and proton beam monitor. We incorporated these into our MIDAS based data acquisition system.

Our DAQ system included an online data quality monitor. This was essential for monitoring beam quality when we requested beam at rates below $10 \mathrm{k}$ protons. The proton beam monitor has also proved to be essential for data analysis since a significant fraction of the data includes either multiple protons per RF bucket or protons in consecutive RF buckets.

We used the facility in several unconventional ways. First, we spent one week in May at the facility setting up our data acquisition system parallel to the current users using raspberry pie boards to emulate our detector signals. Second, we remained in the beam as a tertiary user for three weeks after our allotted time by moving our detector out of the beam during the run time of the primary and secondary users and moving into the beam parasitically while it was not in use. This was typically 4 hours a day in the early morning. We also continued to use the facility after shutdown to test the detectors with ${ }^{90} \mathrm{Sr}$ and ${ }^{55} \mathrm{Fe}$ sources available at the facility.

Primary lessons learned relating to the facility are that online monitoring of beam profiles and instantaneous rates would be very helpful. The slow spill of roughly 4 seconds per minute is very painful during commissioning of the test setup and a shift to something like 1 second, 4 times a minute would be an enormous improvement. The week before the test that we used to set up the DAQ was invaluable and we recommend it to any other users who can do this. The time spent as tertiary user was also invaluable and we would like to use that as a model for performing long term quality control on all our straw planes before they are inserted in the experiment. 
T-1043/Mu2e (E. C. Dukes, A. Artikov, D. Chokheli, R. Ehrlich, K. Francis, M. J. Frank, R. C. Group, S. Hansen, A. Hocker, Y. Oksuzian, P. Rubinov, E. Song, Y. Wu)

Beam used: $120 \mathrm{GeV}$ protons Run dates: $\quad$ 6-MAY-2015 to 12-MAY2015, 1-JUN-2015

Motivation and Goals: Measure photoelectron yields from prototype Mu2e Cosmic Ray Veto scintillation counters.

\section{Setup:}

Counters with integrated readout

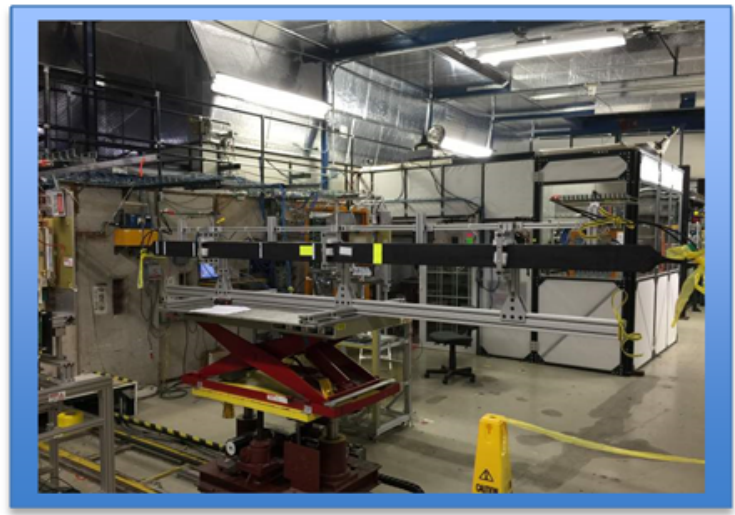

T-1043 setup at FTBF

electronics were installed on a motion table to systematically scan the face of the counters with the beam at varying incidence angles. The FTBF multwire proportional chambers were used to localize the hits with a precision of $\sim 0.25 \mathrm{~mm}$.

\section{Results and Impact:}

Analysis is ongoing of the data. Shown in the figure is a transverse scan of the PE yield (sum of both SiPMs on one end) for two side-byside counters, each of $50 \mathrm{~mm}$ width and $20 \mathrm{~mm}$ thickness. The gap between counters and the effect of the fiber channels are evident. These results and more were presented at DPF2015, and are being written up for NIM.

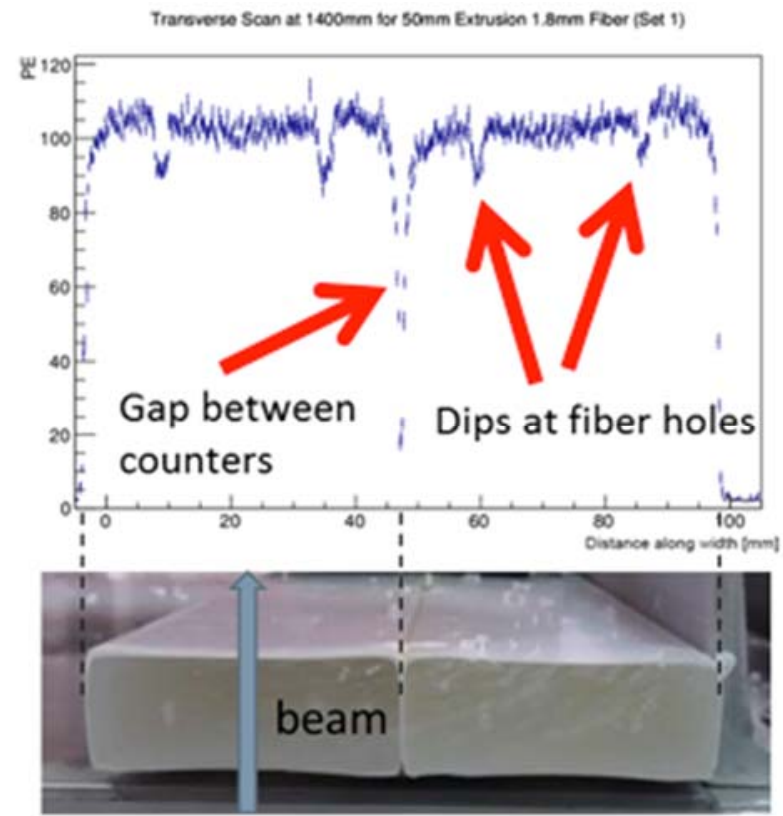


T-1058/New fast calorimeters based on LYSO studied in frame of the T-1058.

(D. Anderson, A. Apresyan, A. Bornheim, J. Duarte, C. Pena, A. Ronzhin, M. Spiropulu, J. Trevor, S. Xie, Caltech)
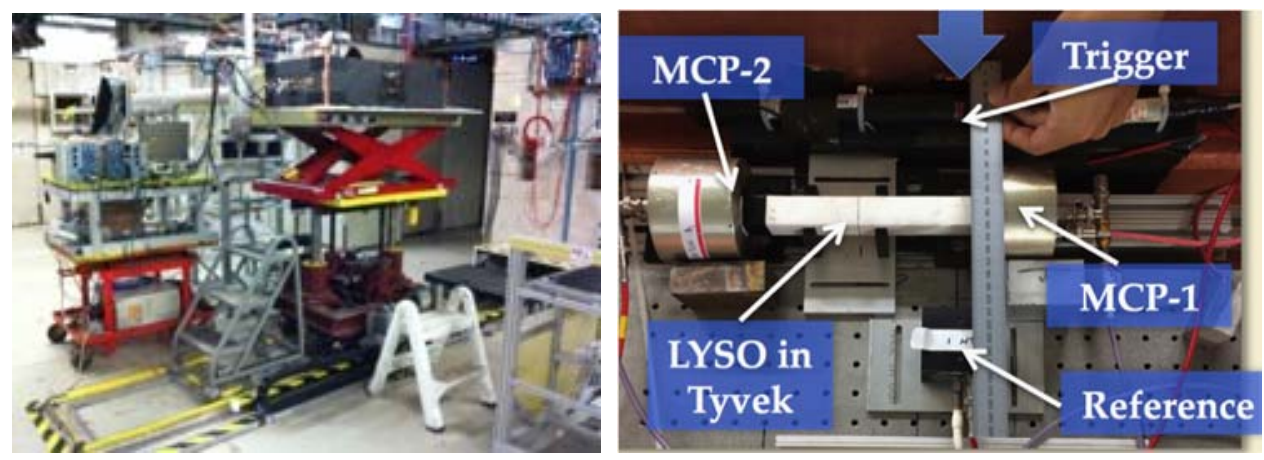

\section{Setup:}

$\mathrm{X}-\mathrm{Y}$ moving table, 6C2.

\section{Beam used:}

4, 8, 16, $32 \mathrm{GeV}$ electrons, $120 \mathrm{GeV}$ protons. Run dates: March 2015

\section{Motivation and Goals:}

Timing properties of LYSO based calorimeter's modules measured on the FTBF beam lines. 30 picosecond of time resolution obtained for full size module. The time resolution (TR) dependent on electron's energy. We have shown that it is possible to make fast and high radiation resistive calorimeter, based on this new approach. Better than 10 ps time resolution obtained for TOF based on Photek 240 MCP-PMTs. The best in the world TOF TR for $41 \mathrm{~mm}$ diameters of the detector's sensitive area.

\section{Part of the obtained results:}
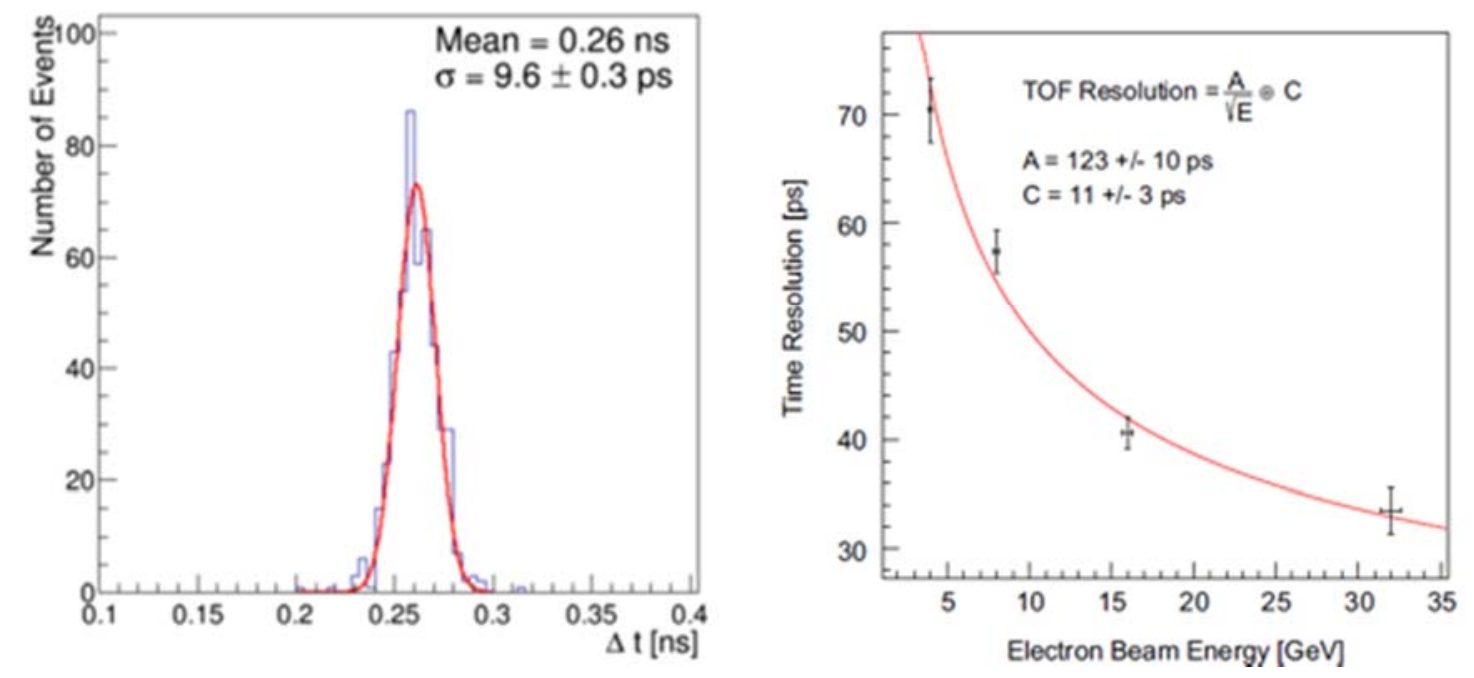


\section{Results and Impact:}

Results published in NIM, On Timing Properties of LYSO-based Calorimeters, D. Anderson, A. Apresyan, A. Bornheim, J. Duarte, C. Pena, A. Ronzhin, M. Spiropulu, J. Trevor, S. Xie, NIM, A794 (2015) 7-14.

Precision timing measurements for high energy photons, Dustin Anderson, Artur Apreysan, Adi Bornheim, Javier Duarte, Harvey Newman, Cristian Pena, Anatoly Ronzhin, Maria Spiropulu, Jason Trevor, Si Xie, Ren-Yuan Zhu, NIM, A787 (2015) 94. 
T-1059/(prototype) optical-TPC (E. Oberla, H. J. Frisch)

Beam used: 8,16, and $32 \mathrm{GeV} / \mathrm{c}$ secondary positive pions on MCenter target

Run dates: 5-March to 20-May 2015

\section{Motivation and Goals:}

The Optical Time Projection Chamber (OTPC) is a small-scale ( $40 \mathrm{~kg}$ ), prototype water Cherenkov detector using a combination of Micro-Channel Plate photo-multipliers (MCP-PMTs) and optical mirrors. The goal is to demonstrate the

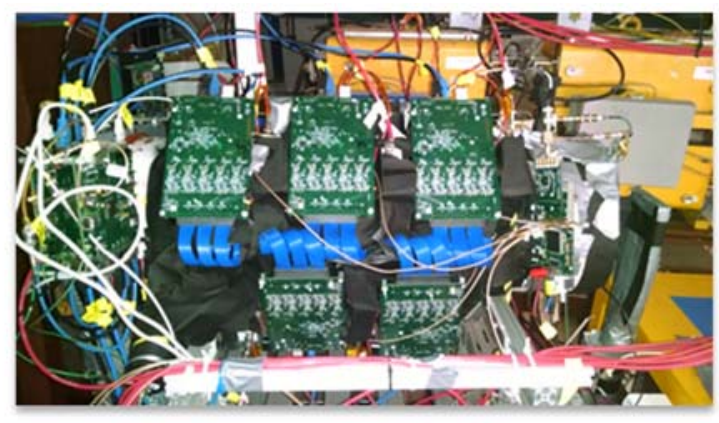

Figure 1: OTPC installed in MCenter along secondary line, behind the $\sim 1 \mathrm{~m}$ thick steel collimator capability of reconstructing the tracks of relativistic particles by sampling the 'drifted' Cherenkov light. (An analog to electrons in a liquid noble TPC, for example.) The MCP-PMTs, with fast waveform digitizing readout, allow for the detection of individual Cherenkov photons in 3D with $\sim 75$ ps timing a $2 \mathrm{D}$ spatial resolution of a few square $\mathrm{mm}$.

\section{Setup:}

The T-1059 setup within the beam-enclosure is shown in Figure 1. The detector volume is an 11 " diameter, 34 " long cylinder filled with deionized water. The center-line of the detector was lined up with the MCenter secondary beam.

We are grateful for the use of a multi-purpose stand on which to mount the detector (constructed by Todd Nebel of the FTBF). High voltage was routed from the enclosure to the MCenter counting room. We took data parasitically to the LArIAT experiment and its beam requests, though we managed a few shifts in which we had primary control.

\section{Results and Impact:}

A first experimental test of tracking, in 3D, muons through a water Cherenkov detector was demonstrated. This technique is applicable to future water Cherenkov neutrino detectors. The analysis and results were presented in a physics Ph.D. dissertation and will be submitted for review and publication in Nuclear Instruments and Methods, A: "The design and performance of a prototype water Cherenkov optical time-projection chamber"

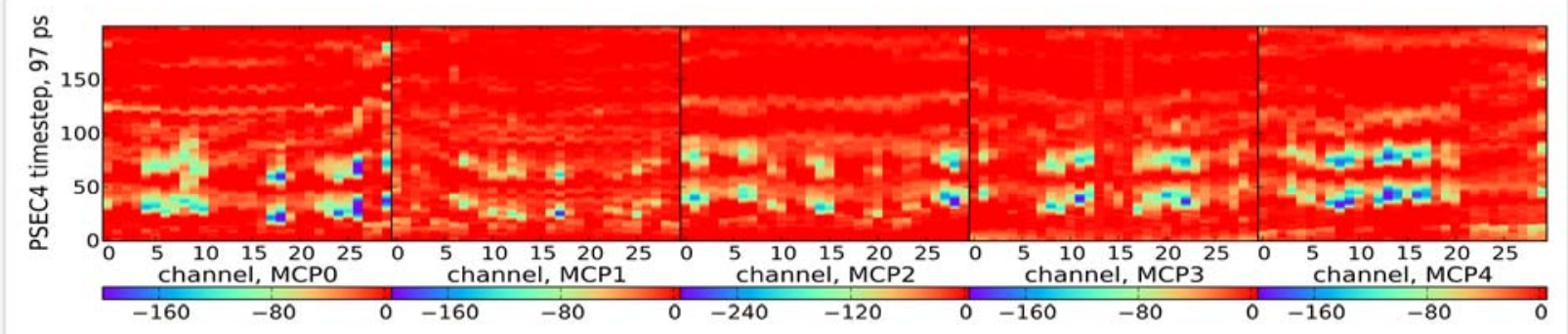

Figure 2: A raw muon track through the prototype OTPC: the time-projection along the beam axis 
T-1064 / STAR Forward Calorimeter System (H. J.Crawford, L. C. Bland, J. M. Engelage, Y. Guo, E. Judd, C. Perkins, Prashanth Shanmuganathan, Zebo Tang, Long Zhou)

Beam used: e-,pi,k,p $2 \mathrm{GeV}$ to $30 \mathrm{GeV}$

Run dates: 12 May - 19 May 2015

Motivation and Goals:

Measure energy resolution and develop electron/hadron identification in pixelized E864 Pb-Scintillating fiber calorimeter for use in DY measurement

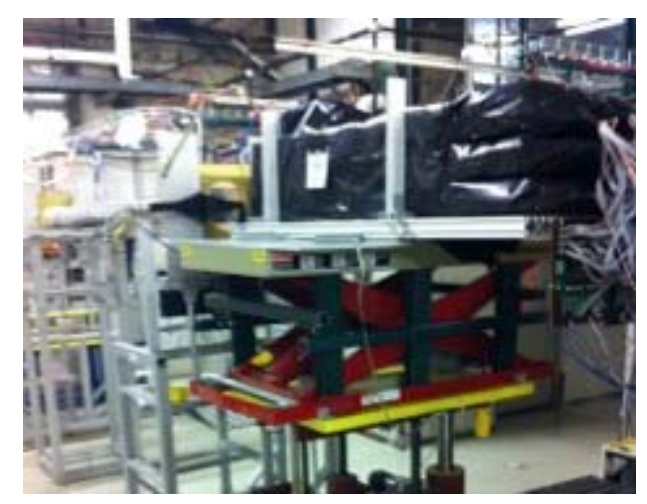

Calorimeter stack for T1064 tests

\section{Setup:}

We staged a stack of 9 cells on the remote controlled table in MT6 area. FNAL provided a modified clock for our daq; scalers to monitor the beam intensities; inner and outer gas $\mathrm{Ck}$ signals, with controls and isolation circuits, which we put in our data stream for PID; MWPCs with controls to verify positions and spot size; collimators in line to control spot size.

\section{Results and Impact:}

Using the different beams and the Ck PID we have shown that the pixelized calorimeter has excellent electron and hadron discrimination capabillity based on the ratio of the highest pixel energy to the energy of its associated cluster as shown in the figure. We are preparing a NIM article on results.

There will be a talk at the DNP meeting in Oct.2015 by P. Shanmugathan. 
FERMILAB-TM- 2615-DI November 2015

T1064, beam energy dependence for $p r, p c=5,5$

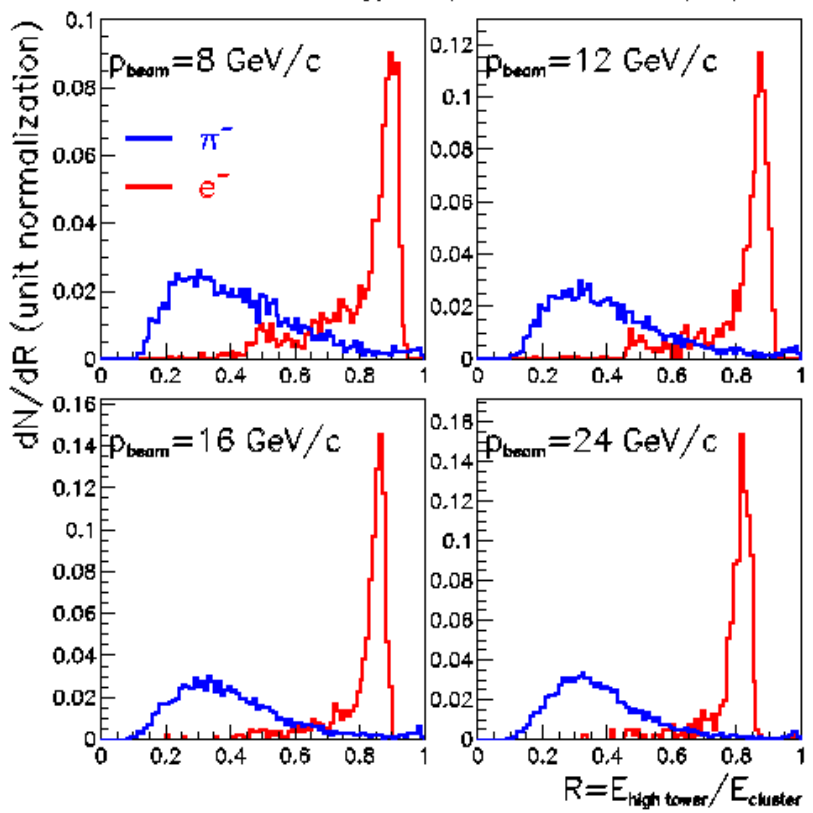

Electron/hadron discrimination from the ratio of the high-tower to the cluster energy. 
T-1065/Secondary emission Calorimeter, (A. Ronzhin, S. Los, E. Ramberg, A. Apresyan, S. Xie, M. Spiropulu, H. Kim)
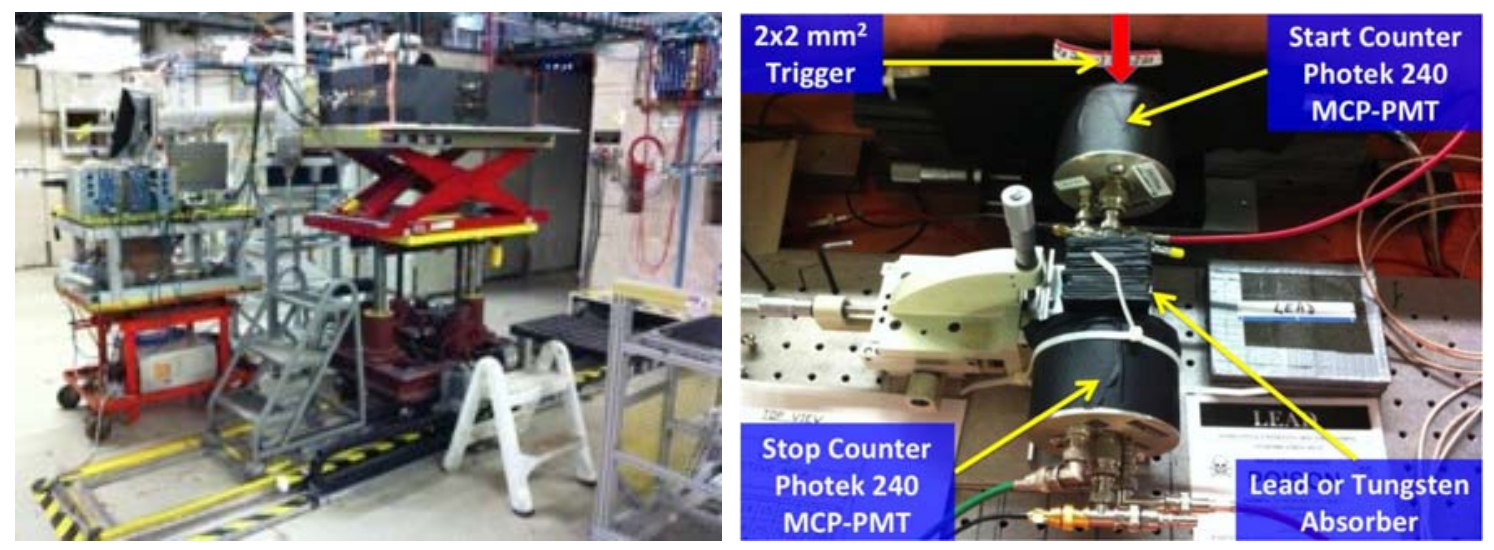

\section{Setup:}

$\mathrm{X}-\mathrm{Y}$ moving table, 6C2.

\section{Beam used:}

8, 16, $32 \mathrm{GeV}$ electrons. March 2015

\section{Motivation and Goals:}

Test of time and position resolution of a brand new approach to make fast, high radiation resistive and low cost calorimeter. The main goal obtained. We have found experimentally that secondary emitters perfectly works as active layer of shower maximum (or a calorimeter layer). Time (TR) and space resolution of the shower maximum (SM) detector is $13 \mathrm{ps}$ and $0.3 \mathrm{~mm}$ (both in $\mathrm{X}$ and $\mathrm{Y}$ directions). The result does not depend on $\mathrm{W}$ or $\mathrm{Pb}$ as absorber material and placement of the active layer in the range of the absorber thickness $2 \mathrm{Xo}-12 \mathrm{Xo}$, Xo is the radiation length. This is the brand new and the best in the world TR so far, obtained in calorimetry.

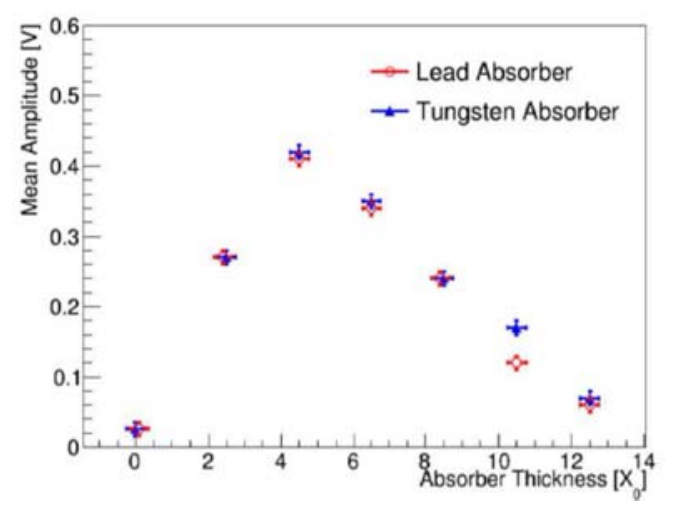

Longitudinal profile of the shower

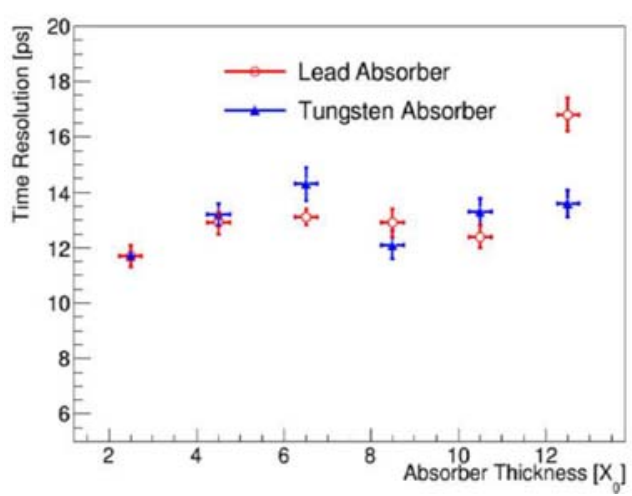

Time resolution of the SM 


\section{Results and Impact:}

Results published in NIM, Direct tests of micro channel plates as the active element of a new shower maximum detector, A. Ronzhin, S. Los, E. Ramberg, A. Apresyan, S. Xie, M. Spiropulu, H. Kim. NIM, A795 (2015), 52-57.

4. Study of the timing performance of micro channel plate photomultiplier

for use as an active layer in a shower maximum detector. A. Ronzhin, S. Los, E. Ramberg, A. Apresyan, S. Xie, M. Spiropulu, H.Kim, NIM, A795 (2015), 288-292. 


\section{Section 3: FY16 Plans}

Currently MTest is set to host 8 experiments for a total of 24 weeks. (Next summer the shutdown is tentatively July - October, 2016.) The experiments are split between general $\mathrm{R} \& \mathrm{D}$, muon physics, collider physics and ion physics (RHIC).

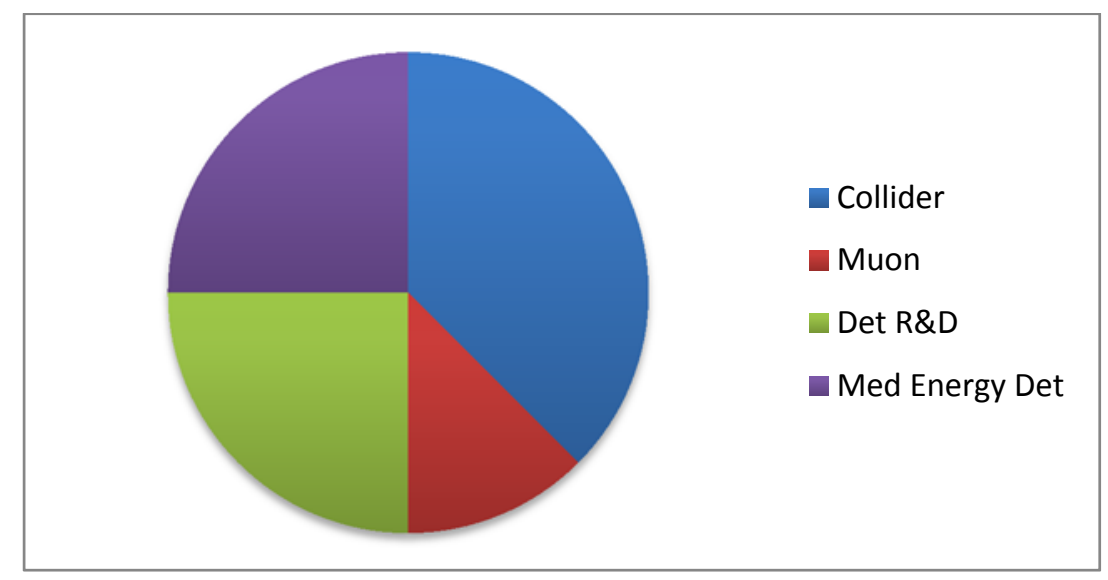

MTest and MCenter beamlines will have a 4 week shutdown Jan 4 - Feb 5 for ICW upgrades to the lab infrastructure. We also plan about 6 weeks of beam studies. The four-week January shutdown will be used to improve the beamline instrumentation.

In MCenter, LArIAT will continue to run. The cryostat is currently open for minor upgrades to the TPC readout electronics and light collection system. The recirculation system for liquid argon will not be installed in MC7 and LArIAT will continue to boil off argon from a dewar. Minor changes are being made to cryogenic system reduce amount of liquid argon used. FY15 included a secondary user. If small, we can continue to have groups in during LArIAT's run in FY16. 